\title{
Journal of Networking and Communication Systems
}

\section{Self-adaptive Particle Swarm Optimization for Optimal Transmit Antenna Selection}

\author{
Nitin Deotale \\ Department of Electronics and Telecommunication Engineering, \\ Priyadarshini College of Engineering, \\ Nagpur, India \\ nitindeotale1502@gmail.com
}

Anuradha Kondelwar

Department of Electronics and Telecommunication Engineering,

Priyadarshini College of Engineering, Nagpur, India

\author{
Uttam Kolekar \\ A.P. Shah College of Engineering, \\ Thane, India
}

Abstract: Generally, Multiple-Input Multiple-Output (MIMO) improves radio communication with enhanced reliability and
capacity. The transmitter and receiver side, since there is an occurrence of multiple antennas, the appropriate Transmit
Antenna Selection (TAS) for obtaining effectual performance is still a difficult point. In this work, a TAS method in the LTE
system by exploiting Self-Adaptive Particle Swarm Optimization (SAPSO) is proposed for enhancing the system
performance. Moreover, it develops self adaptiveness in the PSO by deciding the ability enhancement attained by each
candidate solution for the TAS issue goes after by updating the particle solution on the basis of the enhancement. The
experimentation model considers both Rician and Rayleigh channel, for four antenna configurations such as $2 \times 2,3 \times 2,4 \times 2$
and $4 \times 4$. To the next of the experimentation, it evaluates the performance of SAPSO-TAS with conventional Genetic
Algorithm (GA)-TAS, Artificial Bee Colony (ABC)-TAS, Firefly (FF)-TAS, PSO-TAS, and Grey Wolf Optimization (GWO)-
TAS models.

Keywords: TAS; MIMO; LTE; BER; Rician; Rayleigh

\begin{tabular}{l|l} 
Nomenclature & \\
\hline Abbreviations & Descriptions \\
\hline MIMO & Multiple-Input Multiple-Output \\
GA & Genetic Algorithm \\
PSO & Particle Swarm Optimization \\
AGS & Antenna Group Scheduling \\
FF & Firefly \\
M-MIMO & Massive MIMO \\
GWO & Grey Wolf Optimization \\
CSI & Channel State Information \\
ABC & Artificial Bee Colony \\
AF & Amplify-and-Forward \\
SE & Spectral Efficiency \\
RF & Radio-Frequency \\
EE & Energy Efficiency \\
BFS & Brute Force Search \\
FSO & Free Space Optics \\
SNR & Signal to Noise Ratio \\
EW & Exponential Weibull \\
CDF & Cumulative Distribution Function \\
ABER & Average Bit Error Rate \\
AS & Alamouti Scheme \\
PLS & Physical Layer Security \\
CVTs & Centroidal Voronoit Essellations \\
ASC & Average Secrecy Capacity \\
FDR & Fitness Distance Ratio \\
\hline & \\
\hline
\end{tabular}

(C)Resbee Publishers

https://doi.org/10.46253/jnacs.v3i1.a1 


\begin{tabular}{ll} 
TAS & Transmit Antenna Selection \\
SOP & Secrecy Outage Probability \\
CRN & Cognitive Radio Network \\
PDF & Probability Distribution Function \\
SAPSO & Self-Adaptive Particle Swarm Optimization \\
N-SAW & N Stop and Wait \\
QAM & Quadrature Amplitude Modulation \\
CDF & Cumulative Distribution Function \\
ADC & Analog-to-digital Converter \\
PSO & Particle Swarm Optimization \\
PSM & Precoding-aided Spatial Modulation \\
DASPSM & Deterministic Antenna Selection based PSM \\
ZF & Zero-Forcing \\
ATAS & Adaptive Transmit Antenna Selection \\
RAS & Random Antenna Selection \\
LOS & Line-of-Sight \\
PCA & Principal Component Analysis \\
URA & Uniform Rectangular Antenna Array \\
\hline
\end{tabular}

\section{Introduction}

In a wireless communication system, the error rate performance and the capacity can be considerably enhanced by using a MIMO system. Additionally, it is revealed in Rayleigh fading, the ability of MIMO system raises approximately linearly with the amount of transmit antennas given that there are as a minimum as a lot of receive antennas as channel gain and the transmit antennas among every transmitreceive antenna pair is recognized to the receiver. Between the allegations of MIMO systems are the substantial rise in the hardware cost requisite and the physical restriction of such wireless systems. So, there is a substantial attempt in searching MIMO systems which drastically decrease this complexity, however, they still give the same ability and performance enhancements. A capable approach to attain this objective is to choose a subset of antennas at the receiver and/or transmitter. At the transmitter, antenna selection is considered or at the receiver with complete knowledge of the channel, statistics are considered, however, it is not frequently attained input into practice. Moreover, at the transmitter, the selection of the antenna was considered, for orthogonal space-time block codes at the receiver with exacting emphasis on the Alamouti method was also considered in some work.

The M-MIMO antenna is a multi-user MIMO, which is exploited to particularly create improved employ of spectral competence, sturdiness to interference, augmented bandwidth and minimized latency and it is exploited to offer a solution for maximum demand communication system similar to cellular mobile communication, which needs maximum rate of the data, minimum interference and improved connectivity and coverage. From orthogonal pilot sequences, the channel among the transmitter and receiver is calculated that is inadequate by the consistency channel time. M-MIMO has a few chief problems which are associated with the method exploited in the power allocation and antenna selection. Moreover, a general idea of current development regarding antenna selection and power allocation is provided after that an improvement to the chosen method is provided.

In MIMO systems, antenna selection has been extensively explained as a cost effectual, performanceimproving technology [1]. The cost savings are comprehended by encompassing fewer RF chains than radiating elements at the transmitter and/or receiver. An $\mathrm{RF}$ chain is characteristically a categorization of magnitude, which is a high cost than the radiating element. The $\mathrm{RF}$ chains are switched into the radiating elements on the basis of the (total or incomplete) CSI.

In [9], one more point of attention was studied in that it is accurate transmit-side CSI and a novel method called AGS technique that integrates user scheduling and antenna selection for following an additional minimization in feedback overhead was explained. The AGS was constructed to improve the channel besides choosing the optimal antennas on the basis of the optimal gain attained. The experimentation has exhibited that the proposed method completes an inspiring feedback overhead minimization over the existing beamforming approaches within the similar target sum-rate requirements. In [11] [12], MIMO can get better SE in cooperation with the EE. In both transmitting and receiving end, the antenna selection procedure is necessary for the M-MIMO system. In [10], a few methods PSO, GA, and ABC were exploited and evaluated and they are exploited to attain improved antenna selection with superior ability and minimum CPU time. In [13], it concentrated attention on the CSI, by obtaining the CSI plays a fundamental role to offer maximum system performance. A competent TAS approach attaining the number of the necessary CSI for an M-MIMO system in the broadcast channel was exploited. The technique is to employ receiver selected channel and feedback the chosen to 
the transmitter in the appearance of feedback till each and every receiver terminate subsequently the transmitter will decide that antennas will be exploited and that are to be abandoned. The purpose is to exploit data rates. The best solution is an extremely complex comprehensive BFS [14], overall likely amalgamations of users and antennas.

The main contribution of this paper is to develop a TAS approach for improving system performance. The proposed TAS model is based on the enhanced meta-heuristic search algorithm termed as PSO. Initially, the channel capacity is evaluated via the channel model and it is definite as the objective function for the TAS issue. In Rayleigh fading channel, the chosen objective function states the Ergodic ability of the system, for that precise channel estimate will be used. Next, a self-adaptive PSO presents to resolve the TAS model. In the PSO, the self adaptiveness is initiating by deciding the ability enhancement attained by each particle solution for the TAS problem persuade by updating the particle solution on the basis of the enhancement.

\section{Literature Review}

In 2019, Kehinde O. Odeyemi, Pius A. Owolawi [1], investigated the performance analysis of TAS on a multiuser asymmetric FSO/RF system in widespread order user scheduling. At the destination, a dualhop system with one relay, one source, and multiple FSO users were measured without direct link among the sender and receiver. The RF link was given to FSO and Nakagami-m fading relations go after EW distribution. By employing the AF CSI on the basis of the increase relay protocol, the corresponding CDF end-to-end SNR at the destination was derived and exploited to attain the investigative expression for the system outage probability.

In 2018, Rajiv Kumar and Sudakar Singh Chauhan [2] presented a novel feedback rate competent TAS with AS with vigorous error performance that enhances PLS in the MIMO wiretap channel. In two steps, the developed model was attained the transmitter fixes one antenna and subsequently, the transmitter chooses the powerful antenna beyond the remaining NT ones, based upon the criticism from the receiver. For data transmission, the AS was used at the fixed antennas. Two different cases on the basis of the availability of CSI of eavesdropper at the transmitter were evaluated. The developed model attains superior ASC and worse SOP through choosing two antennas.

In 2017, Saifur Rahman Sabuj and Masanori Hamamura [3], investigated the energy effectiveness and the outage probability in a CRN, modeling the positions of the cognitive users and primary users as a Poisson point procedure. For the energy efficiency and the outage probability, the closed-form expressions with the deliberation of the probabilities of untenanted (not used by the primary users) successful transmission and channel selection for defective recognition in an interference-limited environment of CRN was derived. Moreover, a TAS technique was proposed for the cognitive transmitter in such networks and consequently expands closed-form expressions for the energy efficiency and the outage probability. Here, the study investigated highlights the significance of integrating the abilities of untenanted channel selection and triumphant transmission in CRN to attain optimal performance concerning outage probability and energy effectiveness. With respect to the energy competence, there was an optimal threshold which increases the energy effectiveness.

In 2017, Elizabeth M. Okumu and Mqhele E. Dlodlo [4], developed a study for the performance of TAS at the data link layer in MIMO systems with N-SAW. The effect of packet size, amount of SAW procedures and the packets stalling in the receiver rearranging buffer, because of N-SAW, on TAS was examined. selection of antenna was implemented as a signal processing approach which improves the MIMO system performance; two models, capacity and throughput based TAS were considered.

In 2019, Parvez Shaik et al [5], analyzed the performance of a dual-hop cooperative MIMO system for a variety of innovative QAM models. A non-regenerative MIMO method with the TAS scheme was wellthought-out wherever a single transmit antenna increasing the total received signal power was chosen for transmission. In the developed system model, it was implicit that the relay, sender, and receiver were outfitted with multiple antennas. For average symbol error rate, as a performance measure, widespread expressions for square QAM, hexagonal QAM, 32-cross QAM, and rectangular QAM, were examined.

In 2018, Jinseok Choi and Brian L. Evans [6], analyzed the ergodic rate of single TAS in lowresolution ADC systems. For multiple antenna systems by exploiting low-resolution ADCs was a possible power-minimization solution. In wireless transceivers, minimum-resolution ADC systems with TAS can additionally power utilization and minimize cost. Taking into consideration such systems, the approximated poorer bound of the ergodic rate with TAS was derived. The approximated distribution of the summation of Weibull random variables was used to deal with confronts concerned in evaluating the quantization error. Subsequently, in closed form for a single receive antenna, the approximated ergodic rate with TAS was derived, which shows the TAS gain in minimum-resolution ADC systems. 
In 2019, Yu Huang et al [7], proposed two TAS methods for PSM to improve confidentiality performance with minimized hardware complexity. Using the CSI of both the illegitimate and legitimate associations the initial scheme was determined, called DASPSM that chooses partial transmit antennas to apply ZF precoding for increasing the secrecy rate. The minimum-complexity DAS-PSM condition was shown for practical implementation. Moreover, the second scheme resorts to RAS with the help of fast radio-frequency switching. Diverse from the DAS-PSM, this therefore-named RAS on the basis of the PSM (RAS-PSM) dispenses with the CSI of the wiretap channel that creates the ZF precoding matrix related with arbitrarily chosen to transmit antenna integration during each symbol period, generating extra turbulence which exclusively affects the eavesdropper. To contest the problem of minimum secrecy rate in the Maximum SNR regime because of the finite alphabet inputs, time-varying artificial noise with extra power can be integrated with both aforesaid schemes that were exemplified in the RAS-PSM.

In 2018, Li Zhu et al [8], conducted a study on the point to- point mm-Wave LOS MIMO channel on the basis of the URA. For URA, the deployment of the optimal antenna was derived to attain the utmost multiplexing gain in the mm-Wave LOS MIMO channel. For the URA with fixed parameters, since the distance among receiver and transmitter augments, a few singular values of the channel matrix were inclined to be zero. In this scenario, it was not essential to employ all of the transmit antennas. Hence, in accordance with two types of CSI feedback strategies, two ATAS schemes based on PCA was proposed for this mm-Wave LOS.

\section{Optimal Transmit Antenna Selection for LTE}

\subsection{Transmit Antenna Selection Model}

In Fig 1, the system model of MIMO-OSTBC is demonstrated, which is associated with the feedback for the TAS scenario. Moreover, it is used to the P-ary PSK modulator and the OSTBC encoder encodes the modulated output. In reality, the current simulation is performed for an individual TAS and therefore, a single transmission link is contemplated. Moreover, $\mathrm{N}_{(\mathrm{TA})}$ is represented as the number of transmit antennas associated with the output side of a switch. Generally, an individual antenna is chosen by the switch based on the feedback move up from the receiver. Likewise, $\mathrm{N}_{(\mathrm{RS})}$ is represented as the number of antennas associated with the receiver side. In addition, the channels of the model experience a flat Rayleigh fading environment. The system with no suitable antenna selection is indicated as $\left(\mathrm{N}_{(\mathrm{TA})} ; \mathrm{N}_{(\mathrm{RS})}\right)$.

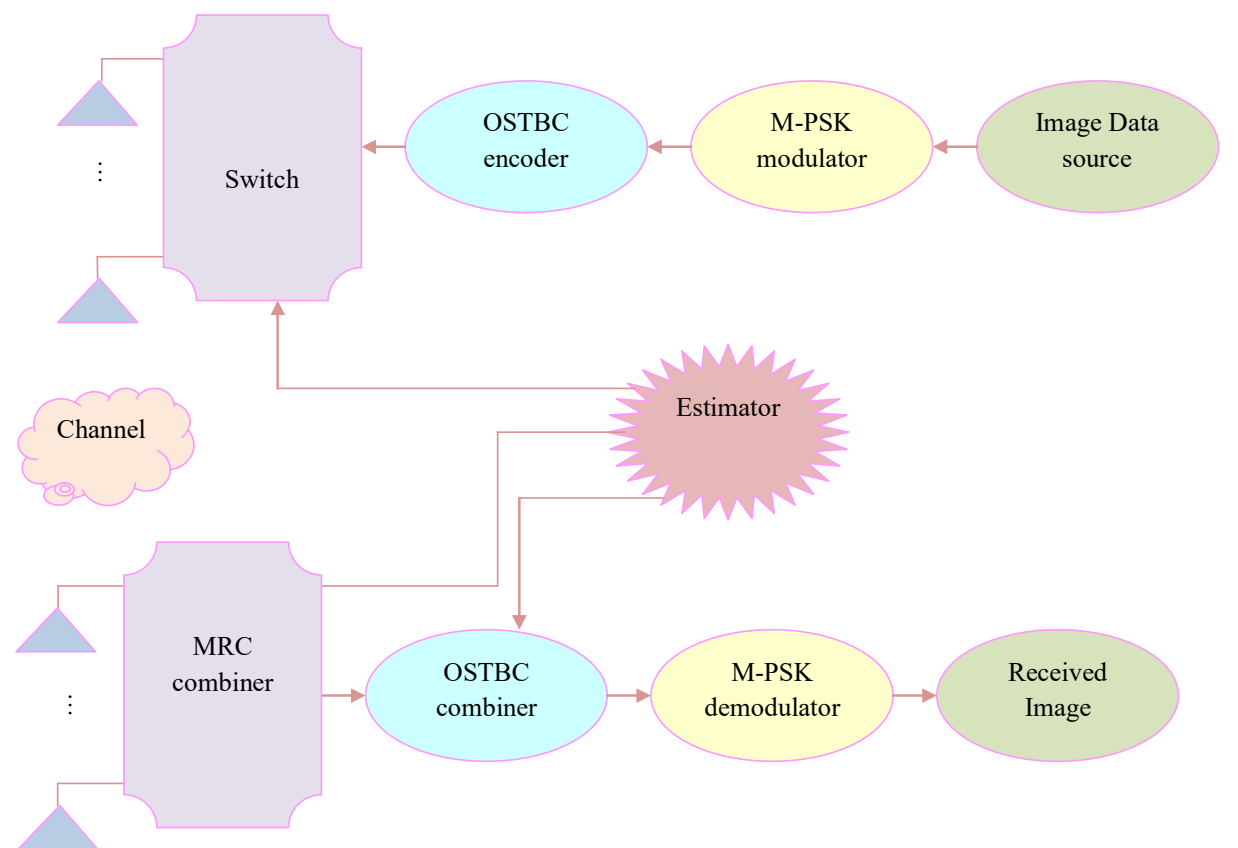

Fig. 1. Block diagram of TAS model

Consider P as the channel matrix, that has a dimension $\left(\mathrm{N}_{(\mathrm{TA})} \times \mathrm{N}_{(\mathrm{RS})}\right)$ and $\mathrm{p}_{\mathrm{i}, \mathrm{j}}$ as the channel fading coefficients, where $i=1,2 \ldots \mathrm{N}_{(\mathrm{TA})}$ and $\mathrm{j}=1,2 \ldots \mathrm{N}_{(\mathrm{RS})}$. The fading coefficients aforesaid are the patterns of 
Rayleigh fading operation. In addition, the column vector of the form $\left(\mathrm{N}_{(\mathrm{TA})} \times 1\right)$ indicates the channel among individual transmit antenna and $\mathrm{N}_{(\mathrm{RS})}$ receive antennas. The channel is supposed to be constant, and this particular constant value within the section might change autonomously for a transmission section. Actually, the constant value can be the consolidation of constant gain and constant phase of the channel.

Eq. (1) indicates the received signal vector model, Y for the transmitted signal b over the antenna that is chosen as a single for a particular time instant $t$. Moreover, $\mathrm{E}_{\mathrm{b}}$ shows the symbol energy, $\mathrm{b}$ denotes the signal vector of OSTBC encoder $\mathrm{G}$ indicates the number of selected antenna subsets, $\mathrm{P}_{\mathrm{ml}}$ indicates the matrix of channel gain, $\mathrm{n}^{\text {(vector) }}$ denotes the noise vector and mldenotes the index of $1^{\text {th }}$ chosen column matrix.

$$
\mathrm{Y}=\sqrt{\frac{\mathrm{E}_{\mathrm{b}}}{\mathrm{G}}} \mathrm{M}_{\mathrm{ml}} \mathrm{b}+\mathrm{n}^{\text {(vector })}
$$

\subsection{Constraints of Transmit Antenna Selection}

To measure the channel capacity, it is necessary to transmit the pilot signal from each transmit antennas $\mathrm{N}_{(\mathrm{TA})}$ towards the complete receive antennas $\mathrm{N}_{(\mathrm{RS})}$. Hence, eq. (2) denotes the channel capacity for each path. The covariance matrix with the size $(\mathrm{N} \times \mathrm{N})$ stated in eq. $(2), \mathrm{C}_{\mathrm{P}}$ indicates the Hermitian matrix and $\mathrm{I}_{\mathrm{m}}$ indicates the identity matrix.

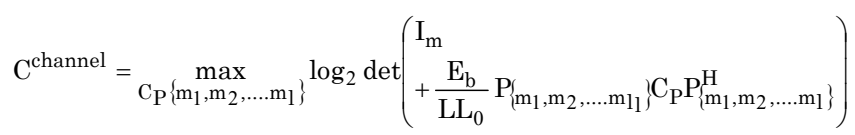

Let an equal power is assigned for every channel in the model (on the basis of the Rayleigh fading channel) consequently that the covariance matrix is equivalent to the identity matrix $\mathrm{C}_{\mathrm{P}}=\mathrm{I}_{\mathrm{L}}$. Since the number of antennae selected here is single, the value of $\mathrm{I}_{\mathrm{L}}=1$. Therefore, eq. (3) signifies the improved format of eq. (2), whereas i signifies the selected subset of antenna for the transmission principle.

$$
\mathrm{C}^{\text {channel }}=\max _{\mathrm{C}_{\mathrm{P}}\left\{\mathrm{m}_{1}, \mathrm{~m}_{2}, \ldots \mathrm{m}_{1}\right\}} \log _{2} \operatorname{det}\left(\mathrm{I}_{\mathrm{d}} \mathrm{E}_{\mathrm{b}} \mathrm{E}_{\mathrm{LL}_{0}} \mathrm{P}_{\left\{\mathrm{m}_{1}, \mathrm{~m}_{2}, \ldots \mathrm{m}_{1}\right\}} \mathrm{C}_{\mathrm{P}} \mathrm{P}_{\left\{\mathrm{m}_{1}, \mathrm{~m}_{2}, \ldots \mathrm{m}_{1}\right\}}^{\mathrm{H}}\right)
$$

As exhibited in eq. (3), it is necessary to concentrate only on the single subset for every transmit antennas for the calculation of channel capacity. Generally, the channel capacity is an arbitrary variable $1 \leq \mathrm{i} \leq \mathrm{N}_{(\mathrm{RS})}$ and it must be organized in ascending format of magnitude, whereas $\mathrm{C}_{(1)}^{\text {channel }} \leq \mathrm{C}_{(2)}^{\text {channel }} \leq \ldots \mathrm{C}_{\mathrm{N}_{(\mathrm{RS})}}^{\text {channel }}$. The selected transmit antenna for transmission stands for $\mathrm{C}_{\mathrm{N}_{(\mathrm{RS})}}$ that is on the basis of the eq. (3).

As the system model is on the basis of the Rayleigh fading channel, eq. (4) indicates the PDF of the Rayleigh distribution. The corresponding equation $\mathrm{b}$ signifies the envelope of the electric field sample and $\Omega$ signifies the product of the quadrature and in-phase component of the electric field.

$$
\mathrm{M}_{\mathrm{R}}(\mathrm{a})=\frac{2 \mathrm{a}}{\Omega} \mathrm{e}^{\frac{-\mathrm{a}^{2}}{\Omega}}, \mathrm{a} \geq 0
$$

Similarly, eq. (5) signifies the CDF for Rayleigh distribution, where $\Omega=2 \sigma^{2}=\mathrm{E}\left[\mathrm{a}^{2}\right]$.

$$
\mathrm{D}(\mathrm{a})=1-\mathrm{e}^{\frac{-\mathrm{a}^{2}}{\Omega}}
$$

In this work, the selection of transmit antennas is only considered. The system can be referred to as $\left(\mathrm{N}_{(\mathrm{TA})}: 1 ; \mathrm{N}_{(\mathrm{RS})}\right)$ an arrangement if a single antenna is chosen from the $\mathrm{N}_{(\mathrm{TA})}$ number of transmits antennas at a time instant $t$. There are two scenarios adaptable to $\left(\mathrm{N}_{(\mathrm{TA})}: 1 ; \mathrm{N}_{(\mathrm{RS})}\right)$ the combo, like $(3: 1 ; 2)$ and $(4: 1 ; 2)$ correspondingly. In this simulation, the $\left(\mathrm{N}_{(\mathrm{TA})}: 1 ; \mathrm{N}_{(\mathrm{RS})}\right)$ integration is considered for the Rayleigh fading channel with the Ergodic capacity principle, which is done for with and without the TAS scenario. In addition, for image data, it estimates the BER analysis for various MIMO configurations.

\section{Optimal Rank Based Transmit Antenna selection for LTE}

\subsection{Objective Function}


The proposed TAS model exploits an understood process of choosing the optimal transmit antenna configuration. In accordance with the process, two objective functions are utilized. The main objective function is an implied function although it chooses the antenna configuration which holds a minimum average as stated in eq. (6). In addition, $\mathrm{RK}_{\mathrm{t}}^{\mu}$ signifies the average rank of $\mathrm{t}^{\text {th }}$ antenna configuration that is stated in eq. (6), whereas $\mathrm{RK}_{\mathrm{t}}^{\mathrm{I}}$ signifies the rank of $\mathrm{t}^{\text {th }}$ antenna configuration in each iteration.

$$
\begin{aligned}
& \mathrm{T}^{*}=\underset{[\mathrm{T}]}{\arg \min } \mathrm{RK}_{\mathrm{t}}^{\mu} \\
& \mathrm{RK}_{\mathrm{t}}^{\mathrm{P}}=\frac{1}{\mathrm{I}^{\max }} \sum_{\mathrm{i}=1}^{\mathrm{I}^{\mathrm{max}}} \mathrm{RK}_{\mathrm{t}}^{\mathrm{I}}
\end{aligned}
$$

Rank Description: $\mathrm{RK}_{\mathrm{t}}^{\mathrm{I}}$ is indicated as the location of probability $\mathrm{PR}\left(\mathrm{B}_{\mathrm{t}}\right)$ as stated in eq. (8) between the BER of every the antenna configurations, whereas $\mathrm{N}_{c}$ signifies the number of antenna configurations and $\mathrm{BR}_{\mathrm{t}}$ signifies the BER of $\mathrm{t}^{\mathrm{th}}$ antenna configuration.

$$
\operatorname{PR}\left(\mathrm{BR}_{\mathrm{t}}\right)=\frac{\mathrm{BR}_{\mathrm{t}}}{\sum_{\mathrm{t}=1}^{\mathrm{N}_{\mathrm{c}}} \mathrm{BR}_{\mathrm{t}}}
$$

The next objective function is the explicit objective function, which plays a key task in assigning ranks for antenna configuration on the basis of the error rate of the received and transmitted data. The subsequent objective function is stated in eq. (9) whereas $\mathrm{S}=\left[\mathrm{s}_{1}, \mathrm{~s}_{2}, \ldots . \mathrm{s}_{\mathrm{N}}\right]$ signifies the number of subcarrier symbols.

$$
\mathrm{S}^{*}=\underset{[\mathrm{S}]}{\arg \min }\left(\frac{1}{\max \left(\mathrm{BR}_{\mathrm{t}}\right)}\right)
$$

\subsection{Self-adaptive PSO Algorithm}

By utilizing the PSO algorithm, an optimization problem is assumed to search a particle in the solution space [15]. Every particle has a velocity and location, whereas the location stands for a value in the solution space and the velocity decides the search direction for the then step. Each particle fitness value decides whether the current searched location, which is superior to others. In the solution space, the entire particles search subsequent to the current optimal particle until all the particles converge to the optimal solution area.

In the conventional PSO approach [16], the solution space dimension is supposed as D, and the

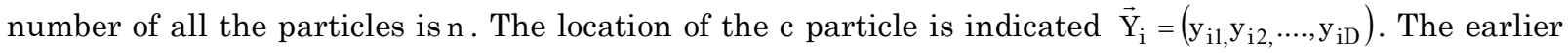
optimal location of the $i^{\text {th }}$ particle is indicated as $\vec{P}_{i}=\left(p_{i 1}, p_{i 2}, \ldots, p_{i D}\right)$, and the earlier optimal location of


time $t$, then the location and velocity of the $i^{\text {th }}$ particle at a time $t+1$ is indicated in eq. (10).

$$
\left\{\begin{array}{l}
u_{i d}(t+1)=w(t) u_{\text {id }}(t)+c_{1} r_{1}\left[p_{\text {id }}(t)-y_{\text {id }}(t)\right]+c_{2} r_{2}\left[p_{k d}(t)-y_{\text {id }}(t)\right], 1 \leq i \leq n, 1 \leq d \leq D \\
y_{\text {id }}(t+1)=y_{\text {id }}(t)+u_{\text {id }}(t+1)
\end{array}\right.
$$

In eq. (10), $c_{1}, c_{2}$ indicates the positive constants named acceleration factors, $w$ indicates the inertia weight factor, and $r_{1}, r_{2}$ indicates arbitrary numbers in the domain $(0,1)$.

The convergence rate of the conventional PSO approach is given to numerous factors, like the unique locations of the particles. For example, if original particles are located near the local extrema, the convergence rate will be minimized clearly. To enhance the global searching ability and convergence rate, a few enhancements are used to the conventional PSO approach. At first, the CVTs approach [17] is proposed to distribute original locations of the particles further dispersedly. Next, a second-order oscillating scheme [18] is exploited to update the locations and velocities of the particles. Further, an FDR item [17] is augmented to the velocity updating equation. The enhanced PSO approach is called the Self-adaptive PSO approach. Fig. 2 exhibits the process model of Self adaptive PSO algorithm.

The CVTs improvement is carried out by the modus operandi as below:

a) Suppose a point group $\mathrm{H}:\left(\hat{\mathrm{h}}_{\mathrm{i}}\right)_{\mathrm{i}=1}^{\mathrm{k}}$, in that the points stand for the initial locations of the particles;

b) Initialize an array js with g elements, and each element is subjected to an initial value of 1 ; 
c) Generate a point $\overrightarrow{\mathrm{m}}$ arbitrarily, and discover the adjacent point $\overrightarrow{\mathrm{h}}_{\mathrm{i}}$ from $\vec{m}$ in group H;

d) As exhibited in eq. (11) execute the loop for 20 iterations.

$$
\begin{gathered}
\overrightarrow{\mathrm{h}}_{\mathrm{j}}=\frac{\overrightarrow{\mathrm{h}}_{\mathrm{j}} \mathrm{js}(\mathrm{j})+\overrightarrow{\mathrm{m}}}{\mathrm{js}(\mathrm{j})+1} \\
\mathrm{js}(\mathrm{j})=\mathrm{js}(\mathrm{j})+1
\end{gathered}
$$

The original locations of the particles with CVTs modification evaluated with those without CVTs modification. As shown, the original locations of the particles are produced additional reasonably and dispersedly by developing CVTs techniques that will cause a high convergence rate and enhanced optimization outcomes.

In addition CVTs modification, a second-order oscillating scheme is exploited and an FDR item is augmented for updating locations and velocities of the particles. The enhanced locations and velocities updating equations are as below:

$$
\left\{\begin{aligned}
\mathrm{u}_{i d}(\mathrm{t}+1) & =\mathrm{w}(\mathrm{t}) \mathrm{u}_{\mathrm{id}}(\mathrm{t}) \\
& +\mathrm{c}_{1} \mathrm{r}_{1}\left[\mathrm{p}_{\text {id }}(\mathrm{t})-\left(1+\delta_{1}\right) \mathrm{y}_{\text {id }}(\mathrm{t})+\delta_{1} \mathrm{y}_{\text {id }}(\mathrm{t}-1)\right] \\
& +\mathrm{c}_{2} \mathrm{r}_{2}\left[\mathrm{p}_{\mathrm{kd}}(\mathrm{t})-\left(1+\delta_{2}\right) \mathrm{y}_{\text {id }}(\mathrm{t})+\delta_{2} \mathrm{y}_{\text {id }}(\mathrm{t}-1)\right] \\
& +\mathrm{c}_{3} \mathrm{r}_{3}\left[\mathrm{p}_{\mathrm{kd}}(\mathrm{t})-\left(1+\delta_{3}\right) \mathrm{y}_{\text {id }}(\mathrm{t})+\delta_{3} \mathrm{y}_{\text {id }}(\mathrm{t}-1)\right] \\
1 & \leq \mathrm{i} \leq \mathrm{n}, 1 \leq \mathrm{d} \leq \mathrm{D} \\
\mathrm{y}_{\text {id }}(\mathrm{t}+1) & =\mathrm{y}_{\text {id }}(\mathrm{t})+\mathrm{u}_{\text {id }}(\mathrm{t}+1)
\end{aligned}\right.
$$

In eq. (12), $\delta_{1}, \delta_{2}$ and $\delta_{3}$ represents arbitrary numbers. To create the enhanced PSO approach have a strong global fast convergence rate and searching abilities, $\delta_{1}, \delta_{2}$ and $\delta_{3}$ are allocated as eq. (13).

At the previous phase

$$
\left\{\begin{array}{l}
\delta_{1}<2 \frac{\sqrt{c_{1} r_{1}}-1}{c_{1} r_{1}}, \delta_{2}<2 \frac{\sqrt{c_{2} r_{2}}-1}{c_{2} r_{2}} \\
\delta_{3}<2 \frac{\sqrt{c_{3} r_{3}}-1}{c_{3} r_{3}} \quad \text { At the previous phase } \\
\delta_{1} \geq 2 \frac{\sqrt{c_{1} r_{1}}-1}{c_{1} r_{1}}, \delta_{2} \geq 2 \frac{\sqrt{c_{2} r_{2}}-1}{c_{2} r_{2}} \\
\delta_{3} \geq 2 \frac{\sqrt{c_{3} r_{3}}-1}{c_{3} r_{3}} \quad \text { At the later stage }
\end{array}\right.
$$

$c_{3} r_{3}\left[p_{n d}(t)-\left(1+\delta_{3}\right) y_{\text {id }}(t)+\delta_{3} y_{\text {id }}(t-1)\right]$ is called the FDR item and

$$
\begin{aligned}
& p_{\text {nd }}(t)=p j^{*}, d \\
& j^{*}=\max _{j} \text { FDR } \\
& \text { FDR }=\frac{\operatorname{Fit}\left(\vec{P}_{j}\right)-\operatorname{Fit}\left(\vec{P}_{i}\right)}{\left|P_{j d}-P_{i d}\right|}
\end{aligned}
$$

The FDR value indicates the ratio among the difference of the fitness and the distance from the $i^{\text {th }}$ particle to the $j^{\text {th }}$ particle. By developing the FDR item, the location of the $i^{\text {th }}$ particle is updated to method the $\mathrm{j}^{* \text { th }}$ particle with the utmost FDR in the iteration.

For the principle of balancing local and global searching ability, the inertia weight factor $w$ is set to differ as below:

$$
\mathrm{w}(\mathrm{t})=\frac{\mathrm{T}-\mathrm{t}}{\mathrm{T}}\left(\mathrm{w}_{\text {initial }}-\mathrm{w}_{\text {final }}\right)+\mathrm{w}_{\text {initial }}
$$

In eq. (17), $\mathrm{w}_{\text {initial }}$ indicates the initial inertia weight factor, T indicates the maximum iterations and $\mathrm{w}_{\text {final }}$ indicates the ultimate inertia weight factor at the last iteration. In this paper, $\mathrm{w}_{\text {initial }}$ and $\mathrm{w}_{\text {final }}$ are set to be 0.4 and 0.9 , correspondingly.

Moreover, the location of the particle $\mathrm{y}_{\mathrm{id}}$ is inadequate in the domain $\left[\mathrm{y}_{\mathrm{d} \text { min }}, \mathrm{y}_{\mathrm{d} \max }\right]$ to avoid the particle from running out of the searching space, whilst the velocity of the particle $u_{i d}$ is restricted in the domain $\left[u_{d \text { min }}, u_{d \text { max }}\right]$ to evade the too maximum or too minimum searching velocity. All the particles out of the bounds are accurate. 


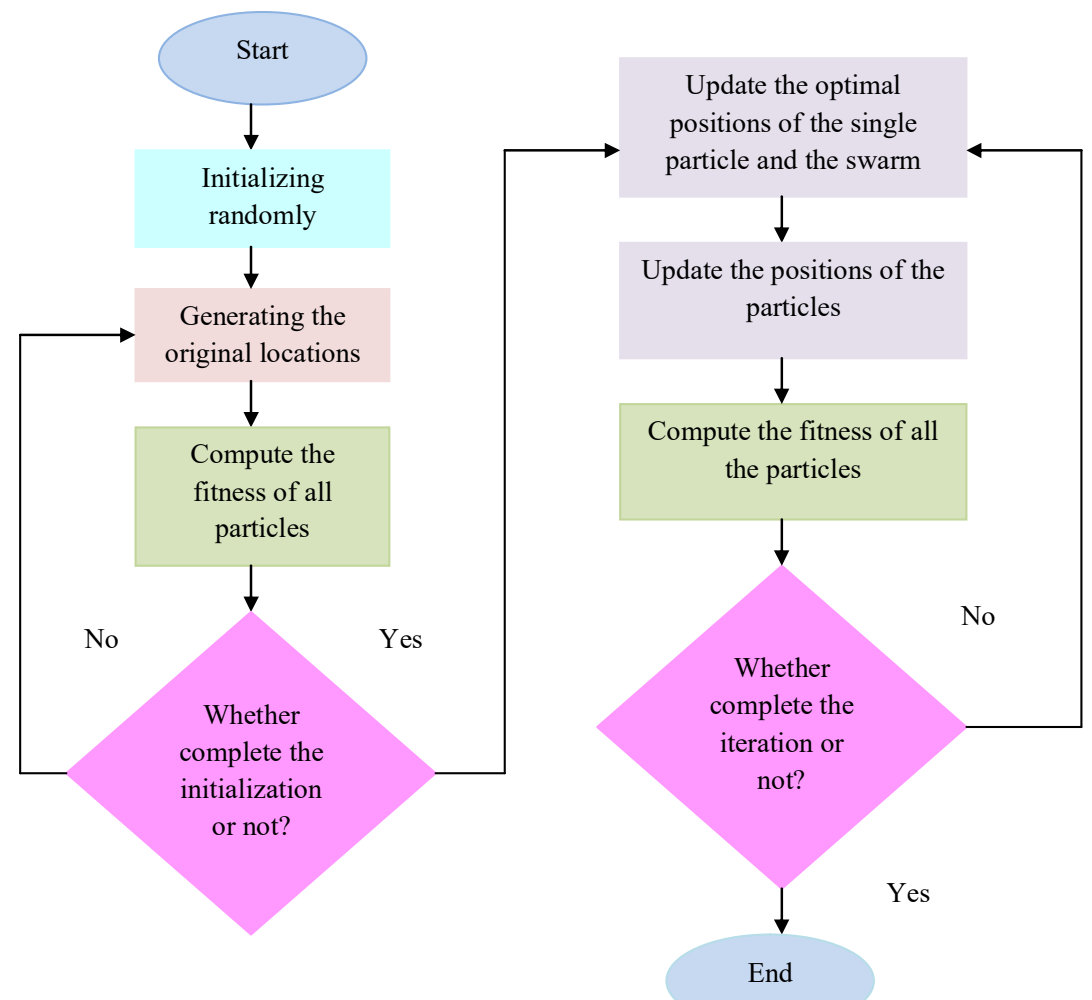

Fig. 2. Flow chart of the Proposed Self-adaptive PSO algorithm

\section{Result and Discussions}

\subsection{Experimental Setup}

In the LTE system, the TAS method exploiting proposed SAPSO experiments in MATLAB, and the outcomes were seen. The particular procedure was performed in both Rician and Rayleigh channels, and it apprehensions 4 antenna configurations such as $2 \times 2,3 \times 2,4 \times 2$ and $4 \times 4$. The results were seen in the mean BER value for each antenna configuration. The experimentation outcomes of the proposed SAPSOTAS were evaluated with the existing methods such as ABC-TAS, FF-TAS [20], GA-TAS, PSO-TAS [21] and GWO-TAS [19] methods.

\subsection{Performance Analysis}

Fig. 3 exhibits the Mean BER analysis of the proposed and existing methods such as ABC-TAS, FF-TAS, GA-TAS, PSO-TAS and GWO-TAS for Rayleigh channels. Here, the proposed methods are 9\% better than ABC-TAS, $5 \%$ better than FF-TAS, $7 \%$ better than GA-TAS, 11\% better than PSO-TAS and 13\% better than GWO-TAS correspondingly. Fig. 4 exhibits the Mean BER analysis of the proposed and existing methods such as ABC-TAS, FF-TAS, GA-TAS, PSO-TAS and GWO-TAS for Rician channels. Here, the proposed methods are $12 \%$ superior to ABC-TAS, $15 \%$ superior to FF-TAS, $16 \%$ superior to GATAS, $18 \%$ superior to PSO-TAS and 15\% superior to GWO-TAS correspondingly. 


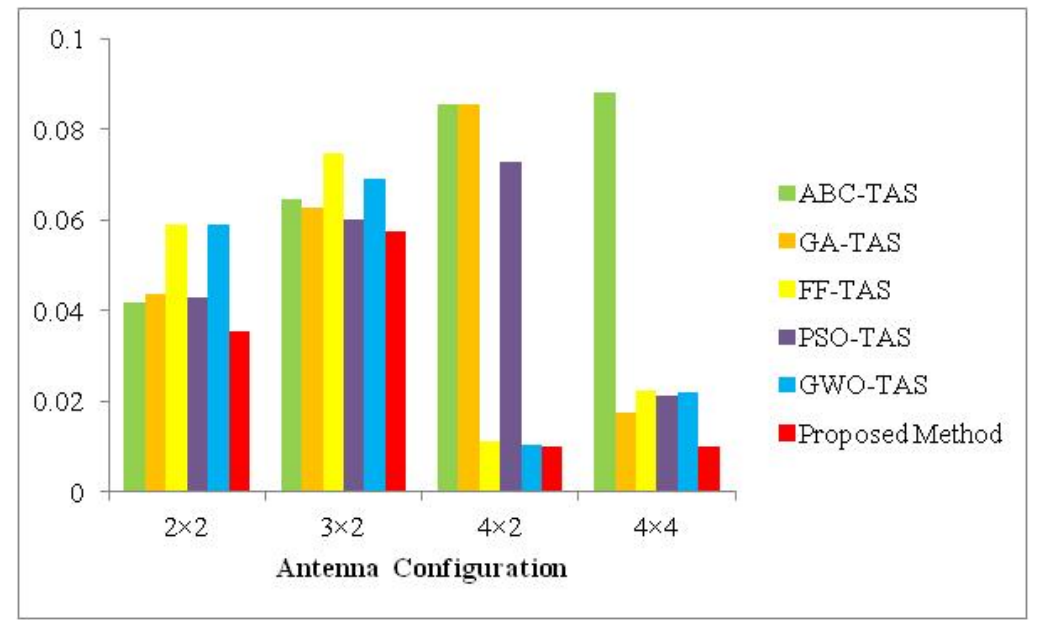

Fig. 3. Mean BER analysis of a proposed and existing models for a Rayleigh channel

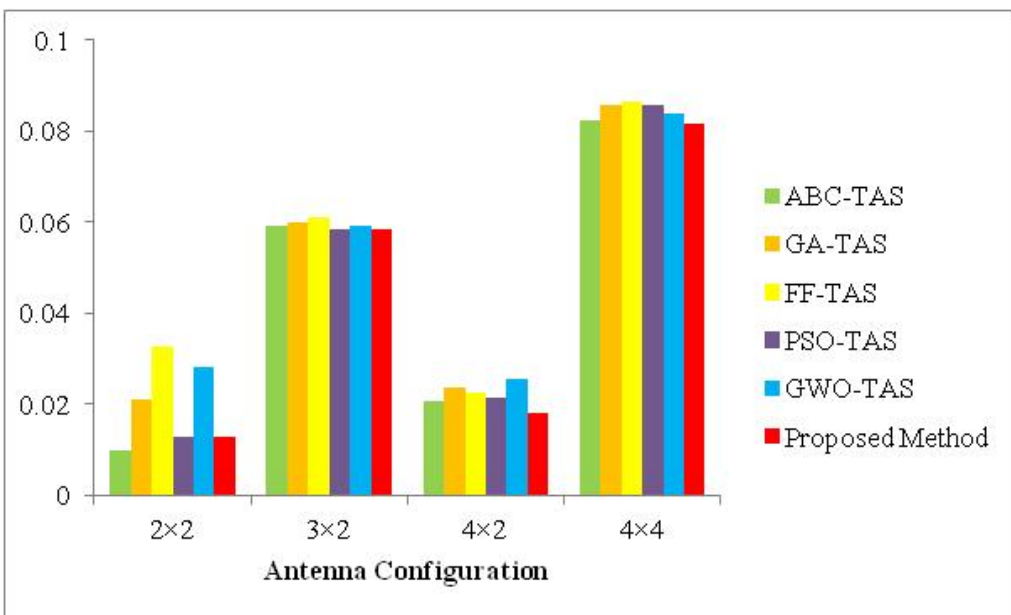

Fig. 4. Mean BER analysis of a proposed and existing models for Rician channel

\section{Conclusion}

In this paper, an optimal TAS approach for enhancing the LTE system performance by exploiting the SAPSO approach was presented. Actually, in the PSO algorithm, self-adaptiveness has proposed by determining the capacity enhancement attained by each particle solution for the TAS problem pursued by updating the particle solution on the basis of the enhancement. The experimentation model has considered both Rician channel and Rayleigh channel, for four antenna configurations such as $2 \times 2,3 \times 2$, $4 \times 2$ and $4 \times 4$. Subsequent to forming the experimentation model, the performance of SAPWO-TAS was evaluated with traditional GA-TAS, ABC-TAS, PSO-TAS FF-TAS, and GWO-TAS models. The examination has measured the BER of the presented and existing models and have confirmed the effectual performance of proposed SAPSO-based TAS model.

\section{Compliance with Ethical Standards}

Conflicts of interest: Authors declared that they have no conflict of interest.

Human participants: The conducted research follows the ethical standards and the authors ensured that they have not conducted any studies with human participants or animals.

\section{References}

[1] Kehinde O. Odeyemi, Pius A. Owolawi, "On the performance of transmit antenna selection in multiuser asymmetric RF/FSO system under generalized order user scheduling",Optik, Volume 197, November 2019. 
[2] Rajiv Kumar, Sudakar Singh Chauhan, "Secrecy analysis of alamouti scheme using feedback-rate efficient transmit antenna selection with robust error performance in the presence of feedback errors", AEU International Journal of Electronics and Communications, Volume 96, November 2018, Pages 40-47.

[3] Saifur Rahman Sabuj, Masanori Hamamura, "Outage and energy-efficiency analysis of cognitive radio networks: A stochastic approach to transmit antenna selection", Pervasive and Mobile Computing, Volume 42, December 2017, Pages 444-469.

[4] Elizabeth M. Okumu, Mqhele E. Dlodlo, "Transmit antenna selection for multiple antenna systems with stall avoidance",Computers \& Electrical Engineering, Volume 58, February 2017, Pages 144-153.

[5] Parvez Shaik, Praveen K. Singya, Vimal Bhatia, "Performance analysis of QAM schemes for non-regenerative cooperative MIMO network with transmit antenna selection", AEU - International Journal of Electronics and Communications, Volume 107, July 2019, Pages 298-306.

[6] J. Choi and B. L. Evans, "Analysis of Ergodic Rate for Transmit Antenna Selection in Low-Resolution ADC Systems," IEEE Transactions on Vehicular Technology, vol. 68, no. 1, pp. 952-956, Jan. 2019.

[7] Y. Huang, M. Wen, B. Zheng, X. Cheng, L. Yang and F. Ji, "Secure Precoding Aided Spatial Modulation via Transmit Antenna Selection," IEEE Transactions on Vehicular Technology, vol. 68, no. 9, pp. 8893-8905, Sept. 2019.

[8] L. Zhu, J. Zhu, S. Wang and J. Zhang, "Adaptive Transmit Antenna Selection Based on PCA for Millimeter Wave LOS MIMO Channel," IEEE Access, vol. 7, pp. 12087-12096, 2019.

[9] HANIF, M., YANG, H. C., BOUDREAU, G., et al. Antenna subset selection for Massive MIMO systems: A tracebased sequential approach for sum rate maximization. Journal of Communications and Networks, 2018, vol. 20, no. 2 , p. $144-155$.

[10] Liu, Z., Du,W., Sun, D. Energy and spectral efficiency trade-off for MassiveMIMOsystems with transmit antenna selection. IEEE Transactions on Vehicular Technology, 2017, vol. 66, no. 5, p. 4453-4457.

[11] QIAN, K., WANG, W. Q., SHAO, H. Low-complexity transmit antenna selection and beamforming for large-scale MIMO communications. International Journal of Antennas and Propagation, 2014, vol. 2014.

[12] Du, L., Li, L., Xu, Y. A genetic antenna selection algorithm with heuristic beamforming for MassiveMIMOsystems. In Proceedings of the IEEE 19th International Symposium on Wireless Personal Multimedia Communications (WPMC). Shenzhen (China), 2016, p. 49-52.

[13] Benmimoune, M., Driouch, E., Ajib, W., et al. Novel transmit antenna selection strategy for Massive MIMO downlink channel. Wireless Networks, 2017, vol. 23, no. 8, 2473-2484.

[14] Dong,Y., Tang,Y., Shenzhen, K. Z. Improved joint antenna selection and user scheduling for Massive MIMO systems. In Proceedings of the IEEE/ACIS 16th International Conference on Computer and Information Science (ICIS). Wuhan (China), 2017, p. 69-74.

[15] Jian Dong, Yujia Xie, Yi Jiang, Fang Liu, Ronghua Shi and Dahong Xiong, "Particle swarm optimization for joint transmit and receive antenna selection in MIMO systems," 2014 IEEE International Conference on Communiction Problem-solving, Beijing, 2014, pp. 237-240.

[16] H. Zhao, H. Long and W. Wang, "WLC06-6: PSO Selection of Surviving Nodes in QRM Detection for MIMO Systems," IEEE Globecom 2006, San Francisco, CA, 2006, pp. 1-5.

[17] X. Jin, G. Sun, Aerodynamic optimization for parameterized wing based on im-proved particle swarm optimization algorithm, Chin. Q. Mech. 33(3) (2012) 461-468.

[18] Y.Y. Wang, B.Q. Zhang, Y.C. Chen, Robust airfoil optimization based on im-proved particle swarm optimization method, Appl. Math. Mech. 32(10) (2011) 1245-1254.

[19] T.C.Subramanyam,S S Tulasi Ram and J B V Subrahmanyam,"HGAGWO: A Multi-Objective Optimal Positioning and Sizing of Fuel Cells in DG Systems"Journal of Computational Mechanics, Power System and Control (JCMPS),Volume 1, Issue 1, October 2018.

[20] Chithra.S and R.Madahana Kumari,"Economic Emission Dispatch in Renewable Energy Systems using FireFly Algorithm",Journal of Computational Mechanics, Power System and Control (JCMPS),Volume 1, Issue 1, October 2018.

[21] Pawan Chandrakant Tapre,Dr. Dharmendra Kumar Singh and Dr. Sudhir R. Paraskar,"HPSOSSA: Enhancement of Dynamic Stability by Optimal Placement of UPFC"Journal of Computational Mechanics, Power System and Control (JCMPS),Volume 2, Issue 2, April 2019. 